Algebraic 83 Geometric $\mathcal{T}$ opology

Volume 4 (2004) 623-645

Published: 23 August 2004

ATG

\title{
Topological Hochschild cohomology and generalized Morita equivalence
}

\begin{abstract}
ANDREW BAKER
ANDREY LAZAREV

Abstract We explore two constructions in homotopy category with algebraic precursors in the theory of noncommutative rings and homological algebra, namely the Hochschild cohomology of ring spectra and Morita theory. The present paper provides an extension of the algebraic theory to include the case when $M$ is not necessarily a progenerator. Our approach is complementary to recent work of Dwyer and Greenlees and of Schwede and Shipley.

A central notion of noncommutative ring theory related to Morita equivalence is that of central separable or Azumaya algebras. For such an Azumaya algebra $A$, its Hochschild cohomology $\operatorname{HH}^{*}(A, A)$ is concentrated in degree 0 and is equal to the center of $A$. We introduce a notion of topological Azumaya algebra and show that in the case when the ground $\mathbb{S}$-algebra $R$ is an Eilenberg-Mac Lane spectrum of a commutative ring this notion specializes to classical Azumaya algebras. A canonical example of a topological Azumaya $R$-algebra is the endomorphism $R$-algebra $\mathrm{F}_{R}(M, M)$ of a finite cell $R$-module. We show that the spectrum of mod 2 topological $K$ theory $K U / 2$ is a nontrivial topological Azumaya algebra over the 2-adic completion of the $K$-theory spectrum $\widehat{K U}_{2}$. This leads to the determination of $\operatorname{THH}(K U / 2, K U / 2)$, the topological Hochschild cohomology of $K U / 2$. As far as we know this is the first calculation of $\operatorname{THH}(A, A)$ for a noncommutative $\mathbb{S}$-algebra $A$.
\end{abstract}

AMS Classification 16E40, 18G60, 55P43; 18G15, 55U99

Keywords $R$-algebra, topological Hochschild cohomology, Morita theory, Azumaya algebra

\section{Introduction}

The recent development of models for stable homotopy category with strictly symmetric monoidal structure has led to the whole new outlook on the relationship between algebra and homotopy theory. In this paper we explore two 
constructions in homotopy category with algebraic precursors in the theory of noncommutative rings and homological algebra, namely the Hochschild cohomology of ring spectra and Morita theory.

Our approach to Morita theory was inspired by the paper of Dwyer and Greenlees [8]. Recall that the classical Morita theorem asserts that the categories of $R$-modules and $\operatorname{End}_{R}(M)$-modules are equivalent, where $R$ is a ring and $M$ is a faithfully projective $R$-module (or, in another terminology, an $R$-progenerator, see [6]). Both the present paper and [8] provide an extension of this theorem to include the case when $M$ is not necessarily a progenerator. Our approach is in some sense complementary to that of Dwyer and Greenlees. Also closely related to our work is that of Schwede and Shipley 23, in which Morita theory for modules over 'ring spectra with several objects' is developed.

One of the central notions of noncommutative ring theory closely related to Morita equivalence is that of a central separable algebra or Azumaya algebra, [1]. A characteristic property of an Azumaya algebra $A$ is that its Hochschild cohomology $\mathrm{HH}^{*}(A, A)$ is concentrated in degree 0 and is equal to the center of $A$. We introduce the notion of a topological Azumaya algebra and show that in the case when the ground $\mathbb{S}$-algebra $R$ is an Eilenberg-Mac Lane spectrum of a commutative ring such algebras specialize to classical Azumaya algebras.

A canonical example of a topological Azumaya $R$-algebra is the endomorphism $R$-algebra $\mathrm{F}_{R}(M, M)$ of a finite cell $R$-module. By analogy with the theory of the Brauer group, it is natural to consider such Azumaya $R$-algebras trivial. We show that $K U / 2$, the spectrum of topological $K$-theory mod 2 is a nontrivial topological Azumaya algebra over $\widehat{K U}_{2}$, the topological $K$-theory spectrum completed at 2 .

This leads to the determination of $\operatorname{THH}(K U / 2, K U / 2)$, the topological Hochschild cohomology of $K U / 2$. As far as we know this is the first calculation of $\operatorname{THH}(A, A)$ for a noncommutative $\mathbb{S}$-algebra $A$. A somewhat surprising outcome is that

$$
\operatorname{THH}(K U / 2, K U / 2) \simeq \widehat{K U}_{2}
$$

We would like to thank John Greenlees and Stefan Schwede for helpful comments.

\section{Notation and conventions}

We choose to work throughout in the category of $\mathbb{S}$-modules of [10], where $\mathbb{S}$ denotes the sphere spectrum. But we note that our constructions should be 
applicable in any version of the underlying model category for spectra with symmetric monoidal structure such as the category of symmetric spectra [14. We will use the symbols $\cong$ and $\simeq$ to denote point-set isomorphism and weak equivalence, respectively. We also use the term $q$-cofibrant in the sense of [10].

If $R$ is an $\mathbb{S}$-algebra, we will often work in the category of left/right $R$-modules $R \mathscr{M} / \mathscr{M}_{R}$ and their derived homotopy categories ${ }_{R} \mathscr{D} / \mathscr{D}_{R}$ as described in [10]. To ease notation, we will usually write $M \wedge N$ for the $R$-smash product $M \wedge_{R} N$, $\mathrm{F}_{R}(M, N)$ or $\mathrm{F}(M, N)$ for the function object $\mathrm{F}_{R} \mathscr{M}(M, N)$ or $\mathrm{F}_{\mathscr{M}_{R}}(M, N)$. We will also write $[M, N]_{*}$ for $[M, N]_{*}^{R}=\pi_{*} \mathrm{~F}_{R}(M, N)$ when it is clear that no ambiguity will result.

We will denote the Spanier-Whitehead dual of an $R$-module $M$ by $M^{*}$, so $M^{*}=\mathrm{F}_{R}(M, R)$.

Following [7, 18, 13, we call an $R$-module $M$ dualizable if it is weakly equivalent to a retract of a finite cell $R$-module. Our justification for this terminology lies in the fact that for objects of $\mathscr{D}_{R}$, the condition of being strongly dualizable in the sense of [7, 18] is equivalent to being a retract of a finite cell $R$-module; see [13, §2], [11, proposition 2.1] and [19. It has been pointed out by the referee that a better word to use might have been 'finite'.

Suppose that $A$ is either an $R$-algebra or an $R$-ring spectrum for a commutative $\mathbb{S}$-algebra $R$. Write $\varphi: A \wedge A \longrightarrow A$ for the product and $\tau: A \wedge A \longrightarrow A \wedge A$ for the switch map. Then we also have the opposite $R$-algebra $A^{\text {op }}$ which consists of the $R$-module $A$ with the product map $\varphi^{\mathrm{op}}=\varphi \circ \tau: A \wedge A \longrightarrow A$. By considering $A$ as a left $A \wedge A^{\mathrm{op}}$-module or a left $A \wedge A^{\mathrm{op}}$-module spectrum, we have the action map in $R \mathscr{M}$

$$
\varphi \circ(\mathrm{id} \wedge \varphi \circ \tau): A \wedge A^{\mathrm{op}} \wedge A \longrightarrow A
$$

which has an adjoint

$$
\mu: A \wedge A^{\mathrm{op}} \longrightarrow \mathrm{F}(A, A) .
$$

This map $\mu$ passes to an adjoint in ${ }_{R} \mathscr{D}$ which we also denote by $\mu$. It is straightforward to check that the following is true.

Lemma 0.1 The action map $\mu: A \wedge A^{\mathrm{op}} \longrightarrow \mathrm{F}(A, A)$ is

- a map of $R$-algebras if $A$ is an $R$-algebra;

- a map of $R$-ring spectra if $A$ is an $R$-ring spectrum.

Let $\mathscr{C}$ and $\mathscr{D}$ be categories. Given two functors $F: \mathscr{C} \longrightarrow \mathscr{D}$ and $G: \mathscr{D} \longrightarrow \mathscr{C}$ we say that $F$ and $G$ form an adjoint pair if $F$ is left adjoint to $G$, or equivalently if $G$ is right adjoint to $F$. We write $\mathscr{C} \simeq \mathscr{D}$ if there is an equivalence $\mathscr{C} \longrightarrow \mathscr{D}$. 


\section{Generalized Morita theory}

In this section we describe a rather general form of Morita theory. Our approach should be compared with that of Dwyer and Greenlees [8]. Here we assume that $R$ is a not necessarily commutative $\mathbb{S}$-algebra and $E$ is a left $R$-module. We have the $\mathbb{S}$-algebra $A=\mathrm{F}_{R}(E, E)$ for which $E$ is a left $A$-module. We will make use of the notions in the following definition, the first of which is perhaps more familiar.

Definition 1.1 Let $E$ be a left $R$-module.

- A right $R$-module $M$ is local with respect to $E$ or $E$-local, if for any $E$-acyclic right $R$-module $X,{ }_{R} \mathscr{D}(X, M)$ is trivial.

- A right $R$-module $N$ is colocal with respect to $E$ or $E$-colocal, if for any $E$-acyclic right $R$-module $Y, R \mathscr{D}(N, Y)$ is trivial.

In the following we will work with both of the categories of left $A$-modules $A \mathscr{M}$ and right $R$-modules $\mathscr{M}_{R}$. Consider the following functors

$$
\begin{aligned}
F_{0}: \mathscr{M}_{R} \longrightarrow{ }_{A} \mathscr{M} ; \quad X \longmapsto X \wedge_{R} E \\
G_{0}:{ }_{A} \mathscr{M} \longrightarrow \mathscr{M}_{R} ; \quad Y \longmapsto \mathrm{F}_{A}(E, Y)
\end{aligned}
$$

It is straightforward to see that $F_{0}$ and $G_{0}$ form an adjoint pair. However, although $F_{0}$ induces a functor $\mathscr{D}_{R} \longrightarrow{ }_{A} \mathscr{D}$, it is not clear that $G_{0}$ induces a functor ${ }_{A} \mathscr{D} \longrightarrow \mathscr{D}_{R}$. To get around this issue, we choose a cofibrant replacement $\widetilde{E}$ for $E$ in $A \mathscr{M}$ and introduce the functors

$$
\begin{aligned}
& F: \mathscr{M}_{R} \longrightarrow{ }_{A} \mathscr{M} ; \quad X \longmapsto X \wedge_{R} \widetilde{E}, \\
& G:{ }_{A} \mathscr{M} \longrightarrow \mathscr{M}_{R} ; \quad Y \longmapsto \mathrm{F}_{A}(\widetilde{E}, Y) .
\end{aligned}
$$

Theorem 1.2 The functors $F$ and $G$ form an adjoint pair. Furthermore, they induce an adjoint pair of functors $\widetilde{F}: \mathscr{D}_{R} \longrightarrow{ }_{A} \mathscr{D}$ and $\widetilde{G}:{ }_{A} \mathscr{D} \longrightarrow \mathscr{D}_{R}$.

If $E$ is a dualizable and $q$-cofibrant left $R$-module, then $\widetilde{F}$ and $\widetilde{G}$ form an adjoint pair of equivalences between the homotopy categories of right $E$-local $R$-modules and left $A$-modules.

Proof The first two statements are immediate. For the remaining part we first need to show that the image of the functor $\widetilde{G}$ lies within the subcategory of $E$ local objects in $\mathscr{D}_{R}$. Let $M$ be an $E$-acyclic right $R$-module, which means that 
$M \wedge_{R} E$ is null in ${ }_{S} \mathscr{D}$ and hence in ${ }_{A} \mathscr{D}$ since ${ }_{A} \mathscr{D}\left(A, M \wedge_{R} E\right) \cong{ }_{S} \mathscr{D}\left(S, M \wedge_{R} E\right)$. We may as well assume that $M$ is $q$-cofibrant in $\mathscr{M}_{R}$. Then

$\mathscr{D}_{R}(M, \widetilde{G}(Y))=\mathscr{D}_{R}\left(M, \mathrm{~F}_{A}(\widetilde{E}, Y)\right)={ }_{A} \mathscr{D}\left(M \wedge_{R} \widetilde{E}, Y\right)={ }_{A} \mathscr{D}\left(M \wedge_{R} E, Y\right)=0$.

We will show that $\widetilde{F} \circ \widetilde{G} \simeq \operatorname{id}_{A} \mathscr{D}$ when $E$ is dualizable. Then $E^{*}=\mathrm{F}_{R} \mathscr{M}(E, R)$ is a right $R$-module for which we choose a cofibrant replacement $W$. Now $\mathrm{F}_{\mathscr{M}_{R}}(W, R) \simeq \widetilde{E}$ in ${ }_{R} \mathscr{M}$, since $E$ is dualizable therein. Then in ${ }_{A} \mathscr{M}$ we have

$$
\begin{aligned}
F \circ G(Y)=\mathrm{F}_{A \mathscr{M}}(\widetilde{E}, Y) \wedge_{R} \widetilde{E} & \simeq \mathrm{F}_{A \mathscr{M}}(\widetilde{E}, Y) \wedge_{R} \mathrm{~F}_{\mathscr{M}_{R}}(W, R) \\
& \simeq \mathrm{F}_{\mathscr{M}_{R}}\left(W, \mathrm{~F}_{A} \mathscr{M}(\widetilde{E}, Y)\right) \\
& \simeq \mathrm{F}_{A \mathscr{M}}\left(W \wedge_{R} \widetilde{E}, Y\right) \\
& \simeq \mathrm{F}_{A} \mathscr{M}\left(W \wedge_{R} E, Y\right) \\
& \simeq \mathrm{F}_{A \mathscr{M}}\left(\mathrm{F}_{R} \mathscr{M}(E, E), Y\right) \\
& \simeq Y .
\end{aligned}
$$

Here we have used the condition that $E$ is $q$-cofibrant in $R \mathscr{M}$ and the evident equivalences of left $A$-modules

$$
A=\mathrm{F}_{R \mathscr{M}}(E, E) \simeq \mathrm{F}_{R} \mathscr{M}(E, R) \wedge_{R} E \simeq W \wedge_{R} \widetilde{E} .
$$

Conversely, let $X$ be an $E$-local right $R$-module. To prove that $\widetilde{G} \circ \widetilde{F}(X) \simeq X$ it is enough to show that $\widetilde{G} \circ \widetilde{F}(X) \wedge_{R} E \simeq X \wedge_{R} E$. But this follows from the previously established fact that $\widetilde{F} \circ \widetilde{G} \cong \mathrm{id}_{A} \mathscr{D}$.

Remark 1.3 Note that in the case when $E$ is a dualizable $q$-cofibrant left $R$ module, the functor $\widetilde{G} \circ \widetilde{F}$ considered as an endofunctor on $\mathscr{D}_{R}$ is isomorphic to the $E$-localization functor $X \mapsto X_{E}$ associating to $X \in \mathscr{D}_{R}$ a left $R$-module $X_{E}$ which is $E$-local and $E$-equivalent to $X$. Indeed, $\widetilde{G} \circ \widetilde{F}$ is $E$-local since the image of $\widetilde{G}$ is $E$-local and the arguments at the end of the proof of Theorem 1.2 show that the natural map $X \longrightarrow \widetilde{G} \circ \widetilde{F}(X)$ is an E-equivalence.

Now consider the following pair of functors:

$$
\begin{aligned}
H:{ }_{A} \mathscr{M} \longrightarrow \mathscr{M}_{R} ; \quad Y \longmapsto \mathrm{F}_{R} \mathscr{M}(E, R) \wedge_{A} Y, \\
F: \mathscr{M}_{R} \longrightarrow A \mathscr{M} ; \quad X \longmapsto X \wedge_{R} E .
\end{aligned}
$$

The functors $H$ and $G$ are not adjoint on the point-set level, however they are becoming such after passing to the derived categories. More precisely we have the following proposition whose proof is analogous to that of Theorem 1.2 and is left to the reader. 
Proposition 1.4 Let $E$ be a dualizable left $R$-module. Then the functors $H$ and $F$ induce functors $\widetilde{H}:{ }_{A} \mathscr{D} \longrightarrow \mathscr{D}_{R}$ and $\widetilde{F}: \mathscr{D}_{R} \longrightarrow{ }_{A} \mathscr{D}$ which form an adjoint pair of equivalences between the homotopy categories of left $A$-modules and right $E$-colocal $R$-modules.

Remark 1.5 Combining Theorem [1.2 with Proposition [1.4 we find that the homotopy categories of right $E$-local and right $E$-colocal $R$-modules are equivalent provided $E$ that is a dualizable right $R$-module. This observation also appears in [13, theorem 3.3.5 (g)].

Our Theorem 1.2 is strikingly similar in formulation and proof to theorem 2.1 of Dwyer and Greenlees [ [8, however it is essentially different. In particular, in their context the appropriate analogues of $E$-local and $E$-acyclic modules are those of E-torsion and E-trivial modules given in the following definition.

Definition 1.6 A left $R$-module $N$ is $E$-trivial if ${ }_{R} \mathscr{D}(E, N)$ is trivial. A left $R$-module $M$ is $E$-torsion if ${ }_{R} \mathscr{D}(M, N)$ is trivial for all $E$-trivial $N$.

For completeness, we reproduce part of [8, theorem 2.1], even though in what follows we stick to the version of Theorem 1.2 .

The notions of $E$-trivial and $E$-torsion left $R$-modules are in some ways dual to $E$-acyclic and $E$-local right $R$-modules. We will make use of the functors

$$
\begin{aligned}
& T: \mathscr{M}_{A} \longrightarrow R \mathscr{M} ; \quad X \longmapsto X \wedge_{A} E, \\
& S: R \mathscr{M} \longrightarrow \mathscr{M}_{A} ; \quad M \longmapsto \mathrm{F}(E, M) .
\end{aligned}
$$

Now we can formulate a theorem which is 'dual' to Theorem 1.2 and appears in 8 .

Theorem 1.7 The functors $T$ and $S$ form an adjoint pair. Furthermore, they induce an adjoint pair of functors $\widetilde{T}:{ }_{A} \mathscr{D} \longrightarrow{ }_{R} \mathscr{D}$ and $\widetilde{S}:{ }_{R} \mathscr{D} \longrightarrow \mathscr{D}_{A}$.

If $E$ is a dualizable and q-cofibrant left $R$-module, then $\widetilde{T}$ and $\widetilde{S}$ form an adjoint pair of equivalences between the homotopy categories of left E-torsion $R$-modules and right $A$-modules.

Remark 1.8 The remaining part of the Dwyer-Greenlees theorem is 'dual' to Proposition 1.4 
We now return to Theorem 1.2, The main advantage of this over the classical Morita theorem (apart from the fact that we are working in the topological framework) is that $E$ is not required to be a generator in ${ }_{R} \mathscr{D}$. We cannot hope that the categories $\mathscr{D}_{R}$ and ${ }_{A} \mathscr{D}$ are equivalent if $E$ is not dualizable. However for certain right $R$-modules $M$ it is still possible that the unit of the adjunction

$$
M \longmapsto \widetilde{G} \widetilde{F}(M)=\mathrm{F}_{A}\left(E, M \wedge_{R} E\right)
$$

is an $E$-equivalence (without the assumption that $E$ is dualizable). We are especially interested when this is the case for $M=R$.

Definition 1.9 For a right $R$-module $M$ define its $E$-completion $E^{\wedge} M$ to be the right $R$-module $\widetilde{G} \widetilde{F}(M)$ in ${ }_{R} \mathscr{D}$.

We now make a few easy but important observations which sometimes help to decide whether the $E$-completion agrees with $E$-localization. Since the functor $\widetilde{G}$ takes its values in the subcategory of $E$-local right $R$-modules the $E$-completion of any right $R$-module will be $E$-local. The unit and counit adjunction morphisms ? $\widetilde{G} \widetilde{F}(?)$ and $\widetilde{F} \widetilde{G}(?) \longmapsto$ ? determine the pair of maps

$$
\left.E \wedge_{R} M=F M \longrightarrow \widetilde{F} \widetilde{G} \widetilde{F} M=E \wedge_{R} E\right\urcorner M \longrightarrow F M=E \wedge_{R} M,
$$

whose composite is obviously the identity map. Therefore the $\mathbb{S}$-module $E \wedge_{R} M$ is a retract of $E \wedge_{R} E \wedge M$. In particular, the $E$-homology of $M$ is a direct summand of the $E$-homology of $E \widehat{\wedge}$. Therefore a map $M \longrightarrow N$ is an $E$ equivalence if and only if the induced map $E^{\wedge} M \longrightarrow E^{\wedge} N$ is an equivalence.

Now observe that for a right $R$-module $M$, the canonical map $M \longrightarrow E^{\wedge} M$ is an $E$-equivalence if and only if the map $E_{*} M \longrightarrow E_{*} E^{\wedge} M$ is an isomorphism, which happens exactly when $E^{\wedge} E^{\wedge} M=E^{\urcorner} M$. In other words, the $E$ completion agrees with $E$-localization in those cases when the $E$-completion is idempotent. The following two purely algebraic examples are instructive.

Example 1.10 Let $R=\mathbb{k}[y]$ be the polynomial algebra over a field $\mathbb{k}$ on one generator $y$ in degree 0 and $E=\mathbb{k}$ considered as a left $R$-module. Then $A=$ $\mathrm{RHom}_{R}(\mathbb{k}, \mathbb{k})$ is easily seen to be $\Lambda(x)$, the exterior algebra on one generator in degree -1 . Indeed, the unit map $\mathbb{k} \longrightarrow \mathbb{k}[y]$ gives rise to an augmentation $A \longrightarrow k$ and it is easy to see that any $\mathbb{k}$-augmented differential graded $\mathbb{k}$ algebra whose homology algebra is $\Lambda(x)$ is formal, i.e. is quasi-isomorphic to its homology. Since $E$ is a dualizable $R$-module we see that the category of $E$-local $R$-modules is equivalent to the category of $A$-modules. The canonical 
map $M \longrightarrow E \wedge M$ is the $E$-localization by Remark 1.3. Letting $M=R$, we see that

$$
R_{E}=E^{\curlywedge} R=\operatorname{RHom}_{\Lambda(x)}(\mathbb{k}, \mathbb{k})=\mathbb{k}[[y]]
$$

Example 1.11 Let $R=\Lambda_{\mathbb{k}}(x)$ to be the exterior algebra over a field $\mathbb{k}$ on one generator of degree -1 and $E=\mathbb{k}$ considered as a left $R$-module. Then $A=\mathrm{RHom}_{R}(\mathbb{k}, \mathbb{k})$ is easily seen to be the power series algebra over $\mathbb{k}$ on one generator of degree 0 . Since $\mathbb{k}$ is not a dualizable $R$-module (it has infinite homological dimension) we cannot conclude that the category of left $R$-modules is equivalent to the category of right $A$-modules. However we can analyze the situation using Theorem [1.7 It is easy to see that the algebra $R=\Lambda_{\mathbb{k}}(x)$ is quasi-isomorphic to $\mathrm{RHom}_{A}(\mathbb{k}, \mathbb{k})$. Clearly the (left) $A$-module $\mathbb{k}$ is dualizable. Therefore the category of $R$-modules is equivalent to the category of torsion $A$-modules. In particular the canonical map $R \longrightarrow E^{\wedge} R$ is an equivalence even though $E$ is not a dualizable $R$-module.

Here are some topological examples suggestive of double centralizer theorems of algebra which we briefly recall, for details see [15, 22].

Let $A$ be a $\mathbb{k}$-algebra over some field $\mathbb{k}$ and let $M$ be a simple right $A$-module. Then $B=\operatorname{End}_{A} M$ acts naturally on the left of $M$ there is a canonical monomorphism $A \longrightarrow B$ which allows us to view $A$ as a $\mathbb{k}$-subalgebra of $B$. Then $\operatorname{End}_{B} M=A$.

In the topological context there are similar phenomena, at least in the homotopy category. Such examples were hinted at by the first author in 2], although the details required further elaboration of the theory of $\mathbb{S}$-algebras and their modules to be made rigorous; they are also related to calculations in [3]. For related ideas also see [9].

Example 1.12 From [12, it is known that for each prime $p$ there is a commutative $\mathbb{S}$-algebra $E_{n}$ whose homotopy ring is

$$
\left(E_{n}\right)_{*}=\pi_{*} E_{n}=\mathbb{W}\left(\mathbb{F}_{p^{n}}\right)\left[\left[u_{1}, \ldots, u_{n-1}\right]\right]\left[u_{n}, u_{n}^{-1}\right],
$$

where $\left|u_{1}\right|=\cdots=\left|u_{n-1}\right|=0,\left|u_{n}\right|=2$ and $\mathbb{W}\left(\mathbb{F}_{p^{n}}\right)$ is the ring of Witt vectors of the finite field $\mathbb{F}_{p^{n}}$. There is also a $E_{n}$-ring spectrum $K_{n}$ with

$$
\left(K_{n}\right)_{*}=\left(E_{n}\right)_{*} /\left(u_{1}, \ldots, u_{n-1}\right)=\mathbb{F}_{p^{n}}\left[u_{n}, u_{n}^{-1}\right] .
$$

Of course, $K_{n}$ is s finite cell $E_{n}$-module. A straightforward calculation with the hypercohomology spectral sequence of [10] yields

$$
\pi_{*} \mathrm{~F}_{E_{n}}\left(K_{n}, K_{n}\right)=\Lambda_{\left(K_{n}\right)_{*}}\left(Q^{0}, \ldots, Q^{n-1}\right),
$$


the exterior algebra over $\left(K_{n}\right)_{*}$ on Bockstein operations $Q^{k}$ of degree 1 associated with the elements $u_{k}$. The spectrum $A_{n}=\mathrm{F}_{E_{n}}\left(K_{n}, K_{n}\right)$ is itself an $E_{n}$-algebra and $K_{n}$ is a left $A_{n}$-module. Another application of the hypercohomology spectral sequence gives a spectral sequence

$$
\mathrm{E}_{2}^{* *}=\operatorname{Ext}_{\left(A_{n}\right)_{*}}^{s t}\left(K_{n}, K_{n}\right) \Longrightarrow \pi_{*} \mathrm{~F}_{A_{n}}\left(K_{n}, K_{n}\right) .
$$

This time we find that

$$
\mathrm{E}_{2}^{* *}=\left(K_{n}\right)_{*}\left[p_{0}, \ldots, p_{n-1}\right]
$$

where $p_{k} \in \mathrm{E}_{2}^{10}$ represents the extension associated with $Q^{k}$. Using Theorem 1.2 we see that $E_{n} \longrightarrow \mathrm{F}_{A_{n}}\left(K_{n}, K_{n}\right)$ is a weak equivalence of $E_{n}$-ring spectra.

Example 1.13 For a prime $p$, the Eilenberg-Mac Lane spectrum $H \mathbb{F}_{p}$ is

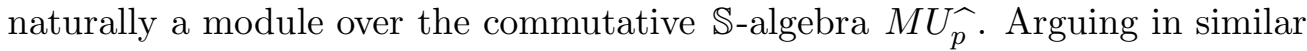

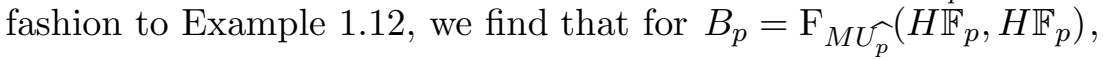

$$
\pi_{*} B_{p}=\Lambda_{\mathbb{F}_{p}}\left(Q^{k}: k \geqslant 0\right),
$$

where $Q^{k}$ is a Bockstein operation associated with a polynomial generator $x_{k}$ for $M U_{*}$ of degree $2 k$ or $x_{0}=p$ when $k=0$. The natural map $M U_{p}^{\prec} \longrightarrow$ $\mathrm{F}_{B_{p}}\left(H \mathbb{F}_{p}, H \mathbb{F}_{p}\right)$ induces a weak equivalence of $M U_{p^{-}}^{- \text {algebras. In this example }}$ we make use of the fact that for the maximal ideal $I=\left(x_{k}: k \geqslant 0\right) \triangleleft M U_{*}$,

$$
\left(M U_{*}\right) \widehat{I}=M U_{* p} .
$$

More generally, using results of [3] it follows that for any ideal $J \triangleleft M U_{*}$ generated by a regular sequence there is an $M U$-module $M U / J$ which is a module over the $M U$-algebra $M U_{J}$. Then for $B_{J}=\mathrm{F}_{M U_{J}}(M U / J, M U / J)$, the natural map

$$
M U_{J} \longrightarrow \mathrm{F}_{B_{J}}(M U / J, M U / J)
$$

is an equivalence of $M U_{J}$-ring spectra. There is also an analogue of this example for the $\mathbb{S}$-algebra $B P$ and its maximal ideal $I_{\infty}=\left(u_{k}: k \geqslant 0\right)$ for a collection of polynomial generators $u_{k} \in B P_{2\left(p^{k}-1\right)}$ and $v_{0}=p$. Under the forgetful map $\mathrm{F}_{B \widehat{P_{p}}}\left(H \mathbb{F}_{p}, H \mathbb{F}_{p}\right) \longrightarrow \mathrm{F}_{S}\left(H \mathbb{F}_{p}, H \mathbb{F}_{p}\right), \pi_{*} \mathrm{~F}_{B P_{p}^{\Im}}\left(H \mathbb{F}_{p}, H \mathbb{F}_{p}\right)$ realizes the Bockstein subalgebra of the usual mod $p$ Steenrod algebra which can be identified with $\pi_{*} \mathrm{~F}_{S}\left(H \mathbb{F}_{p}, H \mathbb{F}_{p}\right)$. 


\section{Azumaya $R$-algebras}

In this section we introduce the notion of topological Azumaya $R$-algebras and prove some of their properties. Azumaya algebras (also known as central separable algebras) have been studied extensively in ring theory, especially in connection with the Brauer group, see for example [1].

We assume that $R$ is a $q$-cofibrant commutative $\mathbb{S}$-algebra. Recall that we usually write $\wedge$ in place of $\wedge_{R}$ and $\mathrm{F}$ in place $\mathrm{F}_{R}$.

Let $A$ be a $q$-cofibrant $R$-algebra and let $\widetilde{A}$ be a $q$-cofibrant replacement for it as an $A \wedge A^{\text {op }}$-module. Then we can form the topological Hochschild cohomology $R$-module of $A$ with coefficients in itself,

$$
\mathrm{THH}_{R}(A, A)=\mathrm{F}_{A \wedge A^{\mathrm{op}}}(\widetilde{A}, \widetilde{A}) .
$$

Clearly $\operatorname{THH}_{R}(A, A)$ is an $R$-algebra with respect to the composition product.

Definition 2.1 An $R$-algebra $A$ is a topological Azumaya algebra provided the following three conditions hold.

(1) $A$ is dualizable as a left $A \wedge A^{\mathrm{op}}$-module.

(2) $A \wedge A^{\text {op }}$ is $A$-local as a left module over itself.

(3) $R \longrightarrow \operatorname{THH}_{R}(A, A)$ is a weak equivalence.

Remark 2.2 Recall that an algebra $A$ over a commutative ring $R$ is an Azumaya algebra if and only if it is $R$-central (this corresponds to our condition (3)) and a finitely generated (this corresponds to our condition (1)) projective generator in the category of left $A \otimes_{R} A^{\text {op }}$-modules (this corresponds to our condition (2)).

Our next result provides a convenient characterization of topological Azumaya algebras. Recall the action map $\mu: A \wedge A^{\text {op }} \longrightarrow \mathrm{F}(A, A)$ of Lemma 0.1 which in this situation is a map of $R$-algebras.

Proposition 2.3 An $R$-algebra $A$ is a topological Azumaya algebra if and only if the following three conditions hold.

(1) $A$ is a dualizable $R$-module.

(2) $R$ is $A$-local as a module over itself.

(3) $\mu: A \wedge A^{\text {op }} \longrightarrow \mathrm{F}(A, A)$ is a weak equivalence. 
Proof Suppose that the three conditions (1),(2),(3) of Proposition 2.3 are satisfied. According to Theorem 1.2 , the categories $A \wedge A^{\text {op }} \mathscr{D} \simeq \mathrm{F}(A, A) \mathscr{D}$ and $\mathscr{D}_{R}$ are equivalent via the functors $\widetilde{F}$ and $\widetilde{G}$ defined by

$$
\widetilde{F}: X \longmapsto X \wedge \widetilde{A}, \quad \widetilde{G}: Y \longmapsto \mathrm{F}_{A \wedge A \text { op }}(\widetilde{A}, Y),
$$

for an $R$-module $X$ and a left $A \wedge A^{\text {op }}$-module $Y$. The image of $R$ under $\widetilde{F}$ is the left $A \wedge A^{\mathrm{op}}$-module $\widetilde{A}$ and it follows that $\widetilde{A}$, and hence $A$, are both dualizable in $A \wedge A^{\mathrm{op}} \mathscr{M}$ since $R$ is dualizable as module over itself.

Denote the $A$-localization of $R$ as an $R$-module by $R_{A}$ and that of $A \wedge A^{\text {op }}$ as an $A \wedge A^{\mathrm{op}}$-module by $\left(A \wedge A^{\mathrm{op}}\right)_{A}$. Now using (3) and Theorem 1.2 we have

$$
\mathrm{THH}_{R}(A, A) \simeq \mathrm{F}_{\mathrm{F}(A, A)}(\widetilde{A}, \widetilde{A}) \simeq R_{A} \simeq R
$$

and

$$
A \wedge A^{\mathrm{op}} \simeq \mathrm{F}(A, A) \simeq \mathrm{F}_{\mathrm{THH}_{R}(A, A)}(\widetilde{A}, \widetilde{A}) \simeq\left(A \wedge A^{\mathrm{op}}\right)_{A} .
$$

This proves that $A$ is an topological Azumaya $R$-algebra.

Conversely, suppose that the conditions (1),(2),(3) of Definition 2.1 are satisfied. By Theorem 1.2, the categories $\mathscr{D}_{A \wedge A^{\text {op }}}$ and $\operatorname{THH}_{R}(A, A) \mathscr{D} \simeq{ }_{R} \mathscr{D}$ are equivalent via the functors $\widetilde{F}$ and $\widetilde{G}$ defined by

$$
\widetilde{F}: X \longmapsto X \wedge_{A \wedge A^{\text {op }}} \widetilde{A}, \quad \widetilde{G}: Y \longmapsto \mathrm{F}(\widetilde{A}, Y) .
$$

Here $X$ is a right $A \wedge A^{\text {op }}$-module and $Y$ is a left $R$-module. The image of $A \wedge A^{\text {op }}$ is the $R$-module $A$ and it follows that $A$ is a dualizable $R$-module. Furthermore

$$
A \wedge A^{\mathrm{op}} \simeq\left(A \wedge A^{\mathrm{op}}\right)_{A} \simeq \mathrm{F}_{\mathrm{THH}_{R}(A, A)}(\widetilde{A}, \widetilde{A}) \simeq \mathrm{F}(A, A) .
$$

Finally we have

$$
R_{A} \simeq \mathrm{F}_{\mathrm{F}(A, A)}(\widetilde{A}, \widetilde{A}) \simeq \mathrm{F}_{A \wedge A \text { op }}(\widetilde{A}, \widetilde{A})=\operatorname{THH}_{R}(A, A) \simeq R .
$$

Remark 2.4 Proposition 2.3 is an analogue of the standard characterization of an Azumaya algebra $A$ over a ring $R$ as an $R$-progenerator for which the natural map $A \otimes_{R} A^{\text {op }} \longrightarrow \operatorname{Hom}_{R}(A, A)$ is an isomorphism.

Remark 2.5 Condition (3) of Proposition 2.3 should not be confused with the standard characterization of a dualizable $R$-module $A$ by the weak equivalence $A \wedge A^{*} \simeq \mathrm{F}(A, A)$. We do not know whether this condition actually implies dualizability. However, dualizability itself certainly does not imply (3) as the case of a homotopy commutative finite cell $R$-algebra makes clear. 
Now let $R=H \mathbb{k}$, the Eilenberg-Mac Lane spectrum corresponding to a commutative ring $\mathbb{k}$. Then the categories of $H \mathbb{k}$-modules and $H \mathbb{k}$-algebras are Quillen equivalent to the categories of complexes of $\mathbb{k}$-modules and differential graded $\mathbb{k}$-algebras respectively. Recall that a perfect complex of $\mathbb{k}$-modules is a finite length complex consisting of finitely generated projective modules. This is an analogue of a dualizable module in the algebraic category of complexes of $\mathbb{k}$-modules. Under the above mentioned equivalence, our definition of a generalized Azumaya algebra specializes to the following.

Definition 2.6 A generalized Azumaya $\mathbb{k}$-algebra is a differential graded $\mathbb{k}$ algebra $A$ such that

(1) $A$ is quasi-isomorphic to a perfect complex of $\mathbb{k}$-modules;

(2) $\mathbb{k}$ is $A$-local as a module over itself;

(3) the canonical map $A \otimes_{\mathbb{k}}^{\mathrm{L}} A^{\text {op }} \longrightarrow \operatorname{RHom}_{\mathbb{k}}(A, A)$ is an isomorphism in the derived category of $\mathbb{k}$-modules.

Recall that the classical Azumaya $\mathbb{k}$-algebra is defined as a $\mathbb{k}$-algebra which is finitely generated and faithfully projective $\mathbb{k}$-module $A$ such that the canonical map $A \otimes_{\mathbb{k}}^{\mathrm{L}} A^{\mathrm{op}} \longrightarrow \operatorname{Hom}_{\mathbb{k}}(A, A)$ is an isomorphism. Then we have the following

Proposition 2.7 Any Azumaya $\mathbb{k}$-algebra is a generalized Azumaya $\mathbb{k}$-algebra which is concentrated in degree zero as a complex. Conversely, any generalized Azumaya $\mathbb{k}$-algebra whose homology is concentrated in degree zero is multiplicatively quasi-isomorphic to an ordinary Azumaya algebra.

Proof The first statement of the proposition is immediate. For the second statement let $A$ be a generalized Azumaya $\mathbb{k}$-algebra such that $H_{*}(A)=H_{0}(A)$. Then clearly $A$ is equivalent to $H_{0}(A)$ in the homotopy category of differential graded algebras over $\mathbb{k}$. Since $A$ is perfect as a $\mathbb{k}$-module it follows that $H_{0}(A)$ is a finitely generated projective $\mathbb{k}$-module. The only thing left to prove is that $H_{0}(A)$ is a faithful $\mathbb{k}$-algebra, that is the unit map $\mathbb{k} \longrightarrow H_{0}(A)$ has zero kernel. Denote this kernel by $I$, so there is a short exact sequence

$$
0 \rightarrow I \longrightarrow \mathbb{k} \longrightarrow H_{0}(A) \rightarrow 0 .
$$

Tensoring this short exact sequence with $H_{0}(A)$ over $\mathbb{k}$ and taking into account that $H_{0}(A)$ is $\mathbb{k}$-projective we obtain the following short exact sequence:

$$
0 \rightarrow I \otimes_{\mathbb{k}} H_{0}(A) \longrightarrow H_{0}(A) \longrightarrow H_{0}(A) \otimes_{\mathbb{k}} H_{0}(A) \rightarrow 0 .
$$


Since $H_{0}(A)$ is a ring the map $H_{0}(A) \longrightarrow H_{0}(A) \otimes_{\mathbb{k}} H_{0}(A)$ is split by the multiplication map and it follows that $I \otimes_{\mathbb{k}} H_{0}(A)=0$. In other words $I$ is $H_{0}(A)$-acyclic, or equivalently $A$-acyclic. However, $\mathbb{k}$ is $A$-local by assumption, which means that there is no nontrivial map into $\mathbb{k}$ from an $A$-acyclic $\mathbb{k}$-module. Therefore $I=0$ and we are done.

In the topological context it seems natural to relax the notion of the Azumaya algebra somewhat.

Definition 2.8 A weak topological Azumaya $R$-algebra, or just a weak $A$ umaya $R$-algebra, is an $R$-algebra $A$ satisfying conditions (1) and (2) of Definition 2.1 and such that the canonical map $R \longrightarrow \operatorname{THH}_{R}(A, A)$ is the $A$ localization map of $R$ as an $R$-module.

In the algebraic situation the analogue of the weak Azumaya $\mathbb{k}$-algebra would be an algebra $A$ which is a finitely generated projective $\mathbb{k}$-module and such that $A$ is an Azumaya algebra over $\mathbb{k} / I$ where, as above, $I$ is the kernel of the unit map $\mathbb{k} \longrightarrow A$.

Now we have the analogue of Proposition 2.3.

Proposition 2.9 An $R$-algebra $A$ is a weak Azumaya algebra if and only if the following two conditions are satisfied.

(1) $A$ is a dualizable $R$-module.

(2) $\mu$ is a weak equivalence.

Proof The proof is almost identical to that of Proposition 2.3. the only new ingredient being the weak equivalence

$$
\mathrm{F}_{R_{A}}(A, A) \cong \mathrm{F}_{R_{A} \wedge A}(A \wedge A, A) \simeq \mathrm{F}_{A}(A \wedge A, A) \cong \mathrm{F}(A, A) .
$$

Remark 2.10 Notice that a weak topological Azumaya $R$-algebra $A$ is a topological Azumaya algebra over $R_{A}$.

A large supply of weak Azumaya algebras is provided by taking endomorphisms of dualizable $R$-modules.

Proposition 2.11 Let $E$ be a dualizable $q$-cofibrant $R$-module and $A=$ $\mathrm{F}(E, E)$. Then $A$ is a weak Azumaya algebra. 
Proof It is clear that $A$ is a dualizable $R$-module. Furthermore $A$ acts canonically on the left on $E$ and therefore $A$ acts on the right on $E^{*}=F(E, R)$. Denote by $\widetilde{A}$ the cofibrant replacement of $A$ as an $R$-algebra and by $\widetilde{E^{*}}$ the cofibrant replacement of the right $\widetilde{A}$-module $E^{*}$. There is a map of $R$-algebras

$$
\widetilde{A}^{\mathrm{op}} \longrightarrow \mathrm{F}\left(\widetilde{E^{*}}, \widetilde{E^{*}}\right)
$$

which is a weak equivalence since $E$ and hence $\widetilde{E^{*}}$ are dualizable $R$-modules. Now the result follows from the following chain of equivalences in the homotopy category of $R$-algebras

$$
\widetilde{A} \wedge \widetilde{A}^{\mathrm{op}} \simeq \mathrm{F}(E, E) \wedge \mathrm{F}\left(\widetilde{E^{*}}, \widetilde{E^{*}}\right) \simeq \mathrm{F}\left(E \wedge \widetilde{E^{*}}, E \wedge \widetilde{E^{*}}\right) \simeq \mathrm{F}(\widetilde{A}, \widetilde{A}) .
$$

We now give a rather general method of constructing nontrivial examples of topological Azumaya algebras. Let $R$ be a commutative $\mathbb{S}$-algebra whose coefficient ring $R_{*}$ is concentrated in even degrees and suppose that $x \in R_{d}$ is not a zero divisor, so $d$ is even. Following Strickland [24, we consider the homotopy fibre sequence of $R$-modules

$$
\Sigma^{d} R \stackrel{x}{\rightarrow} R \stackrel{\rho}{\rightarrow} R / x \stackrel{\beta}{\rightarrow} \Sigma^{d+1} R .
$$

Smashing this on the left with $R / x$ we obtain a homotopy fibre sequence

$$
R / x \wedge \Sigma^{d} R \stackrel{x}{\rightarrow} R / x \wedge R \stackrel{1 \wedge \rho}{\longrightarrow} R / x \wedge R / x .
$$

Since the first map is null this fibre sequence is split. By [24, lemma 3.7], any such splitting determines a unital and associative product $\varphi: R / x \wedge R / x \longrightarrow$ $R / x$, and any other product $\varphi^{\prime}: R / x \wedge R / x \longrightarrow R / x$ is of the form

$$
\varphi^{\prime}=\varphi+u \circ(\beta \wedge \beta)
$$

for a unique element $u \in \pi_{2 d+2} R / x$. If $\tau:(R / x)^{\wedge 2} \longrightarrow(R / x)^{\wedge 2}$ is the switch map, then $\varphi \circ \tau$ is the multiplication opposite to $\varphi$, so

$$
\varphi \circ \tau=\varphi+v \circ(\beta \wedge \beta)
$$

for a unique element $v \in \pi_{2 d+2} R / x$.

Using the Künneth and universal coefficient spectral sequences, it is straightforward to verify that as $R_{*} / x$-algebras,

$$
\pi_{*} R / x \wedge R / x^{\mathrm{op}}=\Lambda_{R_{*} / x}(\alpha), \quad \pi_{*} \mathrm{~F}(R / x, R / x)=\Lambda_{R / x_{*}}(\bar{\beta}),
$$

where $|\alpha|=d+1$ and $|\bar{\beta}|=-d-1$. We need to make an explicit choice of generators $\alpha$ and $\bar{\beta}$. For $\alpha$, consider the cofibre sequence

$$
R \wedge R / x^{\text {op }} \stackrel{\rho \wedge 1}{\longrightarrow} R / x \wedge R / x^{\text {op }} \stackrel{\rho \wedge 1}{\longrightarrow} \Sigma^{d+1} R \wedge R / x^{\text {op }} .
$$


On applying $\pi_{*}$ we obtain a short exact sequence $0=\pi_{d+1} R \wedge R / x^{\mathrm{op}} \longrightarrow \pi_{d+1} R / x \wedge R / x^{\mathrm{op}} \longrightarrow \pi_{d+1} \Sigma^{d+1} R \wedge R / x^{\mathrm{op}}=\pi_{0} R / x \rightarrow 0$ in which $\rho \in \pi_{0} R / x=\pi_{d+1} \Sigma^{d+1} R \wedge R / x^{\text {op }}$ pulls back to a unique element $\alpha \in \pi_{d+1} R / x \wedge R / x^{\mathrm{op}}$. We also take

$$
\bar{\beta}=\rho \circ \beta: R / x \longrightarrow \Sigma^{d+1} R / x .
$$

Since $R / x$ is an $R$-ring spectrum, Lemma 0.1 shows that $\mu: R / x \wedge R / x^{\text {op }} \longrightarrow$ $\mathrm{F}(R / x, R / x)$ is a map of $R$-ring spectra.

Proposition 2.12 We have

$$
\mu_{*} \alpha=v \bar{\beta} \in \pi_{d+1} \mathrm{~F}(R / x, R / x) .
$$

Proof Since $\varphi \circ(\rho \wedge \rho): R \wedge R=R \longrightarrow R / x$ is the unit map, we have

$$
\varphi \circ \tau=\varphi+v \circ(\beta \wedge \beta)=\varphi+v \varphi \circ(\rho \wedge \rho) \circ(\beta \wedge \beta)=\varphi+v \varphi \circ \bar{\beta} \wedge \bar{\beta} .
$$

Now consider the map

$$
\bar{\beta}^{\prime}=\operatorname{id} \wedge \bar{\beta}: R / x \wedge R / x \longrightarrow \Sigma^{d+1} R / x \wedge R / x
$$

and recall that there are non-canonical equivalences of $R$-modules

$$
R / x \wedge R / x \simeq R / x \vee \Sigma^{d+1} R / x, \quad \Sigma^{d+1} R / x \wedge R / x \simeq \Sigma^{d+1} R / x \wedge R / x .
$$

Applying $\pi_{*}$ we obtain an induced map

$$
\bar{\beta}_{*}^{\prime}: \pi_{*} R / x \wedge R / x \longrightarrow \pi_{*} \Sigma^{d+1} R / x \wedge R / x
$$

whose effect we wish to analyze in terms of the associated direct sum decomposition

$$
\pi_{*} R / x \oplus \pi_{*} \Sigma^{d+1} R / x \longrightarrow \pi_{*} \Sigma^{d+1} R / x \oplus \pi_{*} \Sigma^{2 d+2} R / x .
$$

The natural $R_{*}$-module generating sets for the domain and codomain are 1, $\alpha$ and $\Sigma^{d+1} 1, \Sigma^{2 d+2} \alpha$. The following result is independent of the actual choice of splitting made above.

Lemma 2.13 With respect to the above decomposition, $\bar{\beta}_{*}^{\prime}$ satisfies

$$
\bar{\beta}_{*}^{\prime}(1)=0, \quad \bar{\beta}_{*}^{\prime}(\alpha)=\Sigma^{d+1} 1 .
$$


Proof Smashing the fibre sequence of (2.2) with $R / x$ on the left and taking into account that the map $x: \Sigma^{d+1} R / x \longrightarrow R / x$ is homotopic to zero we find that there is a split homotopy fibre sequence

$$
R / x=R \wedge R / x \stackrel{\rho \wedge \mathrm{id}}{\longrightarrow} R / x \wedge R / x \stackrel{\beta \wedge \mathrm{id}}{\longrightarrow} \Sigma^{d+1} R \wedge R / x=\Sigma^{d+1} R / x .
$$

Now the statement of the lemma becomes obvious after we take the composition of $\beta \wedge$ id with the split inclusion

$$
\Sigma^{d+1} R \wedge R / x \stackrel{\rho \wedge \mathrm{id}}{\longrightarrow} \Sigma^{d+1} R / x \wedge R / x .
$$

Using (2.3) we find that

$$
\begin{aligned}
f & =\varphi \circ(\operatorname{id} \wedge \varphi)+\varphi \circ(v \wedge \varphi) \circ(\operatorname{id} \wedge \bar{\beta} \wedge \bar{\beta}) \\
& =\varphi \circ(\operatorname{id} \wedge \varphi)+\varphi \circ\left(v \bar{\beta}_{*} \wedge \bar{\beta}\right) .
\end{aligned}
$$

Furthermore, the image of $\alpha \in \pi_{d+1}\left(R / x \wedge R / x^{\mathrm{op}}\right)$ in $\pi_{-d-1} \mathrm{~F}(R / x, R / x)=$ $\left[R / x, \Sigma^{d+1} R / x\right]$ agrees with the composition

$$
\gamma: R / x=R \wedge R / x \stackrel{\alpha \wedge \mathrm{id}}{\longrightarrow} \Sigma^{d+1} R / x \wedge R / x^{\mathrm{op}} \wedge R / x \stackrel{f}{\rightarrow} \Sigma^{d+1} R / x .
$$

The composite

$$
\Sigma^{d+1} R \stackrel{\alpha}{\rightarrow} R / x \wedge R / x^{\text {op }} \stackrel{\varphi}{\rightarrow} R / x
$$

is trivial for dimensional reasons, so to compute $\gamma$ only the component $\varphi \circ$ $\left(v \bar{\beta}^{\prime} \wedge \bar{\beta}\right)$ of $f$ is required. It follows from Lemma 2.13 that the composition

$$
S^{d+1} \longrightarrow \Sigma^{d+1} R \stackrel{\alpha}{\longrightarrow} \Sigma^{d+1} R / x \wedge R / x^{\mathrm{op}} \stackrel{\bar{\beta}^{\prime}}{\longrightarrow} R / x \wedge R / x^{\mathrm{op}} \stackrel{\varphi}{\rightarrow} \Sigma^{d+1} R / x
$$

is the $(d+1)$-st suspension of the unit map, therefore $\gamma=v \bar{\beta}$.

Corollary 2.14 Suppose that the $R$-ring spectrum $R / x$ is actually an $R$ algebra and the element $v \in \pi_{2 d+2} R / x$ is invertible. Then $R / x$ is a weak Azumaya $R$-algebra.

Proof By Lemma $0.1 \mu: R / x \wedge R / x^{\text {op }} \longrightarrow \mathrm{F}(R / x, R / x)$ is a map of $R$ algebras, while Proposition 2.12 implies that the canonical $R$-algebra map $\mu$ is a weak equivalence. Since $R / x$ is a finite cell $R$-module, the result follows.

Corollary 2.15 $K U / 2$ is a weak Azumaya $K U$-algebra and $v_{1}^{-1} M U / 2$ is a weak Azumaya $M U$-algebra. 
Proof It was proved in [16] that the spectrum $K U / 2$ is a $K U$-algebra and $M U / 2$ is an $M U$-algebra. Therefore our claim follows from Corollary 2.14 and the formula for the commutators in $M U / 2$ and $K U / 2$ given in [24, theorem 2.12].

Remark 2.16 It seems likely that a weak Azumaya $R$ algebra of the form $R / x$ is never isomorphic to an endomorphism algebra of a dualizable $R$-module. Indeed, let us consider the case when the ring $R_{*} / x$ is a graded field (this holds for $R=K U$ and $R / x=K U / 2)$. If $R / x$ were isomorphic to $\mathrm{F}_{R}(M, M)$, where the $R$-module $M$ is dualizable, then being an $R / x$-module, $M$ would split as a wedge of suspensions of $R / x$. But then the hypercohomology spectral sequence

$$
\mathrm{E}_{2}^{* *}=\operatorname{Ext}_{R_{*}}^{* *}\left(M_{*}, M_{*}\right) \Longrightarrow \pi_{*} \mathrm{~F}_{R}(M, M)
$$

clearly collapses and it follows that the graded $R_{*}$-module $\pi_{*} \mathrm{~F}_{R}(M, M)$ contains odd degree elements. However $\pi_{*} \mathrm{~F}_{R}(M, M)=R_{*} / x$, giving a contradiction.

The following conjecture is supported by the algebraic analysis of $A_{\infty}$ structures on quotients $R / x$ due to the second author [17.

Conjecture 2.17 Let $R$ be a commutative $\mathbb{S}$-algebra such that $R_{*}$ is an evenly graded ring and $x \in R_{*}$ is a nonzero divisor. Then any homotopy associative $R$-ring spectrum structure on $R / x$ can be extended to an $R$-algebra structure.

Suppose provisionally that the above conjecture is true. Let $R$ be a commutative $\mathbb{S}$-algebra whose homotopy is concentrated in even degrees with elements $x \in R_{d}, v \in R_{2 d+2}$ for which $x, v$ is a regular sequence in $R_{*}$. In addition we suppose that 2 is invertible in $R_{*}$. Then by [24, $R / x$ has a unique commutative product $\varphi: R / x \wedge R / x \longrightarrow R / x$. Consider a new product on $R / x$, say

$$
\varphi^{\prime}=\varphi+v / 2 \circ(\beta \wedge \beta) .
$$

From [24, Lemma 3.11] it follows that

$$
\varphi^{\prime} \circ \tau=\varphi+v \circ(\beta \wedge \beta) .
$$

By Conjecture 2.17 the homotopy multiplication $\varphi^{\prime}$ can be extended to an $A_{\infty}$ structure. This leads to

Corollary 2.18 If Conjecture 2.17 is true, then the $R$-algebra $v^{-1} R / x$ has a structure of a topological Azumaya $R$-algebra. 


\section{Topological Hochschild cohomology of topological $K$-theory modulo 2}

This section contains our main application of the Morita equivalence which is the computation of the Hochschild cohomology of $K(1)$ at the prime 2 . We remark that McClure and Staffeldt [20] contain related results obtained by different methods.

Let $\widehat{K U}_{2}$ denote the 2 -adic completion of the complex $K$-theory spectrum with homotopy

$$
\pi_{*} \widehat{K U}_{2}=\mathbb{Z}_{2}\left[v_{1}, v_{1}^{-1}\right] .
$$

Since we work in the categories $\mathscr{M}_{\widehat{K U}_{2}}$ and $\mathscr{D}_{\widehat{K U}_{2}}$ and also $\mathscr{M}_{\mathbb{S}}$ and $\mathscr{D}_{\mathbb{S}}$, we include subscripts indicating when smash products, function spectra and topological Hochschild cohomology are taken over $\widehat{K U}_{2}$ and omit them when they are taken over $\mathbb{S}$; thus for example, $\mathrm{THH}=\mathrm{THH}_{\mathbb{S}}$.

Theorem 3.1 At the prime 2, there is a weak equivalence of spectra

$$
\operatorname{THH}(K(1), K(1)) \simeq \widehat{K U}_{2} .
$$

Proof Observe that Corollary 2.15] together with the fact that $K(1)=\widehat{K U}_{2} / 2$ implies that $K(1)$ is a topological Azumaya $\widehat{K U}_{2}$-algebra and therefore

$$
\mathrm{THH}_{\widehat{K U}_{2}}(K(1), K(1)) \simeq \widehat{K U}_{2} .
$$

Next consider the map

$$
l: K(1) \wedge K(1)^{\mathrm{op}} \longrightarrow K(1) \wedge_{\widehat{K U}_{2}} K(1)^{\mathrm{op}}
$$

induced by the unit map $S \longrightarrow \widehat{K U}_{2}$. From [21] we have

$$
K(1)_{*} K(1)^{\mathrm{op}}=K(1)_{*}\left[a_{0}, t_{k}: k \geqslant 1\right] /\left(a_{0}^{2}-t_{1}, v_{1} t_{k}^{2}-v_{1}^{2^{k}} t_{k}: k \geqslant 1\right)
$$

as $K(1)_{*}$-algebras. Furthermore,

$$
K(1)_{*}^{\widehat{K U}_{2}} K(1)^{\mathrm{op}}=\pi_{*} K(1) \wedge_{\widehat{K U}_{2}} K(1)^{\mathrm{op}}=\Lambda_{K(1)_{*}}\left(\tau_{0}\right) .
$$

Lemma 3.2 The map $l_{*}: K(1)_{*} K(1)^{\mathrm{op}} \longrightarrow K(1)_{*}^{\widehat{K U}_{2}} K(1)^{\mathrm{op}}$ is surjective with kernel generated by the elements $t_{k}$. 
Proof First we prove the surjectivity of $l_{*}$. If $l_{*}$ is not surjective then its image is the subring $K(1)_{*}$ of $K(1)_{*}^{\widehat{K U}_{2}} K(1)^{\text {op }}$ and the dual map

$$
\delta: \operatorname{Hom}_{K(1)_{*}}\left(K(1)_{*}^{\widehat{K U}_{2}} K(1)^{\mathrm{op}}, K(1)_{*}\right) \longrightarrow \operatorname{Hom}_{K(1)_{*}}\left(K(1)_{*} K(1)^{\mathrm{op}}, K(1)_{*}\right)
$$

must factor through $\operatorname{Hom}_{K(1)_{*}}\left(K(1)_{*}, K(1)_{*}\right)=K(1)_{*}$. Notice that the map of (3.1) agrees with the forgetful map

$$
\left[K(1)^{\mathrm{op}}, K(1)\right]_{*}^{\widehat{K U}_{2}} \cong[K(1), K(1)]_{*}^{\widehat{K U}_{2}} \longrightarrow\left[K(1)^{\mathrm{op}}, K(1)\right]_{*}=[K(1), K(1)]_{*} .
$$

But clearly the mod 2 Bockstein operation is a self-map of $K(1)$ considered either as an $\mathbb{S}$-module or an $\widehat{K U}_{2}$-module. This shows that the map $\delta$ of (3.1) cannot factor through $K(1)$ and so $l_{*}$ is onto.

Next we check that $l_{*}\left(t_{k}\right)=0$. This is equivalent to asserting that the map $\delta$ of (3.1) factors through

$$
\operatorname{Hom}_{K(1)_{*}}\left(K(1)_{*} K(1)^{\mathrm{op}} \otimes_{\Sigma_{*}} K(1)_{*}, K(1)_{*}\right),
$$

where $\Sigma_{*}$ is the $K(1)_{*}$-subalgebra in $K(1)_{*} K(1)^{\text {op }}$ generated by the $t_{k}$ 's. Since the image of $l_{*}$ is generated by the mod 2 Bockstein operation $Q_{0}$, it suffices to show that $Q_{0}\left(t_{k}\right)=0$ for all $k$. Since $Q_{0}$ is a derivation it follows from the well-known fact that the algebra $\Sigma_{*}$ is semisimple.

Therefore $\operatorname{Ker} l_{*}$ is an ideal containing all of $t_{k}$ 's. It cannot contain $a_{0}$ since in that case $l_{*}$ would not be onto. The only remaining possibility is that $\operatorname{Ker} l_{*}$ is generated by $t_{k}$ 's.

Now consider two spectral sequences

$$
\begin{aligned}
\mathrm{E}(1)_{2}^{* *}=\operatorname{Ext}_{K(1)_{*} K(1)}^{* *}\left(K(1)_{*}, K(1)_{*}\right) & \Longrightarrow \mathrm{THH}^{*}(K(1), K(1)), \\
\mathrm{E}(2)_{2}^{* *}=\operatorname{Ext}_{K(1)_{*}^{* *} \widehat{K U}_{2}}(1) & \left(K(1)_{*}, K(1)_{*}\right) \Longrightarrow \mathrm{THH}_{\widehat{K U}_{2}}^{*}(K(1), K(1)) .
\end{aligned}
$$

It is now immediate that the $\mathrm{E}_{2}$-term of $(\underline{3.3)}$ is

$$
\mathrm{E}(2)_{2}^{* *}=\operatorname{Ext}_{\Lambda_{K(1) *}^{* *}\left(\tau_{0}\right)}^{*}\left(K(1)_{*}, K(1)_{*}\right)=K(1)_{*}[[y]]
$$

where $y$ has bidegree $(1,1)$. Once again using the fact that $\Sigma_{*}$ is semisimple, we obtain the following base change isomorphism

$$
\begin{aligned}
\mathrm{E}(1)_{2}^{* *} & \cong \operatorname{Ext}_{K(1)_{*} K(1) \otimes_{\Sigma_{*}} K(1)_{*}}^{* *}\left(K(1)_{*} \otimes_{\Sigma_{*}} K(1)_{*}, K(1)_{*}\right) \\
& =\operatorname{Ext}_{\Lambda_{K(1)_{*}\left(a_{0}\right)}^{* *}}\left(K(1)_{*}, K(1)_{*}\right) \\
& =K(1)_{*}[[y]] .
\end{aligned}
$$


It follows that the map $l_{*}$ induces an isomorphism between the spectral sequences of (3.2) and (3.3). Therefore

$$
\operatorname{THH}(K(1), K(1)) \simeq \operatorname{THH}_{\widehat{K U}_{2}}(K(1), K(1)) \simeq \widehat{K U}_{2},
$$

giving the result.

It is natural to ask what happens when $p$ is odd. First of all, the answer depends on whether we consider the 2-periodic or the $2(p-1)$-periodic version of $K(1)$. From [4] it is known that the Adams summand $E(1)$ of $K U_{(p)}$ and its $p$-completion $\widehat{E(1)}$ have unique commutative $\mathbb{S}$-algebra structures. The $2(p-1)$-periodic $K(1)$-theory can be shown to be a homotopy commutative $E(1)$-ring spectrum using the methods of [25, 10, 24]. Since the action map $\mu: K(1) \wedge_{E(1)} K(1) \longrightarrow \mathrm{F}_{E(1)}(K(1), K(1))$ has a homotopy factorization through the augmentation map to $K(1)$, it is not an equivalence and therefore $K(1)$ cannot be a topological Azumaya algebra. On the other hand, if we considers the 2-periodic theory then there exists a homotopy associative multiplication on $K(1)$ for which the canonical map $K(1) \wedge_{K U} K(1)^{\mathrm{op}} \longrightarrow$ $\mathrm{F}_{K U}(K(1), K(1))$ is a weak equivalence. If Conjecture 2.17 were true then in this case we would also have $\operatorname{THH}(K(1), K(1)) \simeq \widehat{K U}_{2}$. To summarize, it seems likely that for an odd prime $p$ the result depends heavily on what $\mathbb{S}$-algebra structure is chosen on $K(1)$. This naturally leads to

Problem 3.3 For an odd prime $p$, classify non-isomorphic $\mathbb{S}$-algebra or $\widehat{K U}_{p^{-}}$ algebra structures on the spectrum $K(1)$.

To illustrate the subtlety of this problem consider again the case $p=2$. We claim that as an $\widehat{K U}_{2}$-algebra $K(1)$ is not isomorphic to its opposite $K(1)^{\text {op }}$. To see this, consider the forgetful map

$$
\mu: K(1)_{*} K(1) \longrightarrow K(1)_{*}^{\widehat{K U}_{2}} K(1) .
$$

By lemma 8 of [21, we know that $K(1)_{*} K(1)=\Sigma_{*}\left[a_{0}\right] /\left(a_{0}^{2}-t_{1}-v_{1}\right)$ and arguments similar to the ones used in the proof of Theorem 3.1 show that $K(1)_{*}^{\widehat{K U}_{2}} K(1) \cong K(1)_{*}\left[\tau_{0}\right] /\left(\tau_{0}^{2}-v_{1}\right)$ and the map $\mu$ of (3.4) is onto, with kernel generated by the elements $t_{i}$.

If $K(1)$ were isomorphic to $K(1)^{\text {op }}$ as an $\widehat{K U}_{2}$-algebra, then the $K(1)_{*}$-algebras $K(1)_{*}^{\widehat{K U}_{2}} K(1)$ and $K(1)_{*}^{\widehat{K U}_{2}} K(1)^{\text {op }}$ would be isomorphic. But this is clearly false since $K(1)_{*}^{\widehat{K U}_{2}} K(1)^{\mathrm{op}}$ contains an exterior generator while $K(1)_{*}^{\widehat{K U}_{2}} K(1)$ does not. 
In effect the above argument shows that $K(1)$ and $K(1)^{\text {op }}$ are not isomorphic even as $\widehat{K U}_{2}$-ring spectra. On the other hand, by [21, lemma 9], the ring spectrum $K(1)$ possesses a nontrivial anti-involution from which it follows that $K(1)$ is isomorphic to $K(1)^{\text {op }}$ as a ring spectrum. We are led to make the following conjecture.

Conjecture 3.4 For $p=2$, there are precisely two structures of an $\widehat{K U}_{2}$ algebra on $K(1)$ and these are opposite to each other. Moreover, the spectrum $K(1)$ possesses a unique structure of an $\mathbb{S}$-algebra.

Note that this conjecture implies that the $\widehat{K U}_{2}$-algebra structure on $K(1) \wedge_{\widehat{K U}_{2}}$ $K(1)^{\text {op }}$ does not depend on the choice of the $\widehat{K U}_{2}$-algebra structure on $K(1)$. Thus $\mathrm{THH}_{\widehat{K U}_{2}}(K(1), K(1))$ is also independent of this choice. This is in agreement with Theorem 3.1

\section{$4 \quad$ Future directions}

Perhaps one of the most interesting ramifications of our constructions lies in the theory of the Brauer group. The classical Brauer group of a ring $R$ is formed by classes of Morita equivalent Azumaya algebras over $R$. Thus, endomorphism rings of finitely generated projective modules are considered trivial in the Brauer group. Our version of Morita theory suggests considering the topological Brauer group of an $\mathbb{S}$-algebra $R$ as generated by topological Azumaya $R$-algebras modulo the endomorphism algebras of dualizable $R$-modules (this yields a set since equivalence classes of retracts of finite cell $R$-modules clearly form a set and so equivalence classes of algebra structures on them also do). Note that for any finite cell $R$-modules $A, B$, there is a weak equivalence

$$
\mathrm{F}_{R}(A, A) \wedge \mathrm{F}_{R}(B, B) \simeq \mathrm{F}_{R}(A \wedge B, A \wedge B) .
$$

This implies that the set of endomorphism algebras of finite cell $R$-modules is closed with respect to smash products and so it forms a submonoid inside the monoid of all Azumaya algebras.

In this sense, the $\widehat{K U}_{2}$-algebra $K U / 2$ gives a nontrivial example of a topological Azumaya algebra, see Remark 2.16. We intend to return to this topic in future work; it seems likely there are connections with the Galois theory of commutative $\mathbb{S}$-algebras initiated by John Rognes, see [5].

We note that all of these constructions also make sense in the category of differential graded modules over a fixed commutative ring. This extends the notion 
of the Brauer group from the purely algebraic context. There are examples of nontrivial generalized Azumaya algebras which are not Azumaya algebras in the classical sense. We restrict ourselves with giving one example; a more detailed discussion can be found in [17. This example provides an algebraic analogue of the $\widehat{K U}_{p}$-algebra $K U / p$; however, in distinction to the topological case, it does not occur for the prime 2 .

Example 4.1 Let $R=\mathbb{Z}\left[v, v^{-1}\right]$ be the ring of Laurent polynomials in one variable $v$ of degree 2 and let $p$ be an odd prime. Consider the differential graded algebra $A$ over $R$ with a single generator $y$ in degree 1 subject to the relation $y^{2}=v$ and whose differential given by $d y=p$. Then

$$
A \stackrel{\mathrm{L}}{\otimes} A^{\mathrm{op}} \simeq \operatorname{RHom}_{R}(A, A),
$$

and furthermore $A$ is clearly dualizable as an $R$-module and therefore $A$ is a (weak) Azumaya $R$-algebra.

\section{References}

[1] M. Auslander and O. Goldman, The Brauer group of a commutative ring, Trans. Amer. Math. Soc. 97 (1960), 367-409. MathReview

[2] A. Baker, $A_{\infty}$ stuctures on some spectra related to Morava $K$-theory, Quart. J. Math. Oxf. 42 (1991), 403-419. MathReview

[3] A. Baker and A. Lazarev, On the Adams spectral sequence for $R$-modules, Algebr. Geom. Topol. 1 (2001), 173-199. MathReview

[4] A. Baker and B. Richter, $\Gamma$-cohomology of rings of numerical polynomials and $E_{\infty}$ structures on $K$-theory, Glasgow University Mathematics Department preprint 02/50. arXiv:math.AT/0304473

[5] A. Baker and B. Richter, Realizability of algebraic Galois extensions by strictly commutative ring spectra, Glasgow University Mathematics Department preprint no. 04/31. arXiv:math.AT/0406314

[6] H. Bass, Algebraic $K$-theory, Benjamin, NY, Amsterdam (1968). MathReview

[7] A. Dold and D. Puppe, Duality, trace, and transfer, Proceedings of the International Conference on Geometric Topology (Warsaw, 1978), PWN (1980), 81-102; also appeared in Proc. Steklov Inst. Math. 154 (1985), 85-103. MathReview

[8] W. Dwyer and J. Greenlees, Complete modules and torsion modules, Amer. J. Math. 124 (2002), 199-220. MathReview

[9] W. Dwyer, J. Greenlees and S. Iyengar, Duality in algebra and topology, preprint (2002): http://wWw.shef.ac.uk/ pm1jg/preprints.html 
[10] A. Elmendorf, I. Kriz, M. Mandell and J. P. May, Rings, modules, and algebras in stable homotopy theory, Mathematical Surveys and Monographs 47, Amer. Math. Soc. (1997). MathReview

[11] H. Fausk, L. G. Lewis and J. P. May, The Picard group of equivariant stable homotopy theory, Adv. in Math. 163 (2001), 17-33. MathReview

[12] P. G. Goerss and M. J. Hopkins, Realizing commutative ring spectra as $E_{\infty}$ ring spectra, preprint (2004): http://www.math.northwestern.edu/ pgoerss/

[13] M. Hovey, J. Palmieri and N. Strickland, Axiomatic stable homotopy theory, Mem. Amer. Math. Soc. 128 (1997). MathReview

[14] M. Hovey, B. Shipley and J. Smith, Symmetric spectra, J. Amer. Math. Soc. 13 (2000), 149-208. MathReview

[15] S. Lang, Algebra, 3rd edition, Grad. Texts in Math. 211, Springer-Verlag (2002). MathReview

[16] A. Lazarev, Towers of $M U$-algebras and the generalized Hopkins-Miller theorem, Proc. London Math. Soc. 87 (2003), 498-522. MathReview

[17] A. Lazarev, Hochschild cohomology and moduli spaces of strongly homotopy associative algebras, Homology Homotopy Appl. 5 (2003), 73-100. MathReview

[18] L. G. Lewis, J. P. May and M. Steinberger, Equivariant stable homotopy theory, Lecture Notes in Mathematics 1213, Springer-Verlag (1986). MathReview

[19] J. P. May, Equivariant Homotopy and Cohomology theories, CBMS Regional Conference Series in Mathematics 91, Amer. Math. Soc. (1996). MathReview

[20] J. E. McClure and R. E. Staffeldt, On the topological Hochschild homology of bu, I, Amer. J. Math. 115 (1993), 1-45. MathReview

[21] C. Nassau, On the structure of $P(n)_{*} P(n)$ for $p=2$, Trans. Amer. Math. Soc. 354 (2002), 1749-1757. MathReview

[22] R. S. Pierce, Associative Algebras, Grad. Texts in Math. 88, Springer-Verlag (1982). MathReview

[23] S. Schwede and B. Shipley, Stable model categories are categories of modules, Topology 42 (2003), 103-153. MathReview

[24] N. Strickland, Products on $M U$-modules, Trans. Amer. Math. Soc. 351 (1999), 2569-2606. MathReview

[25] U. Würgler, Morava $K$-theories: a survey. In: Algebraic Topology Pozań 1989, Lecture Notes in Math. 1474, Springer-Verlag (1991), 111-138. MathReview

Mathematics Department, Glasgow University, Glasgow G12 8QW, Scotland

Mathematics Department, Bristol University, Bristol, BS8 1TW, England.

Email: a.baker@maths.gla.ac.uk and a.lazarev@bristol.ac.uk

URL: http://www.maths.gla.ac.uk/ ${ }^{\sim}$ ajb/ and http://www2.maths.bris.ac.uk/ maxal/

Received: 6 February 2004 Revised: 23 June 2004 